PROCEEDINGS OF THE

AMERICAN MATHEMATICAL SOCIETY

Volume 136, Number 12, December 2008, Pages 4355-4364

S 0002-9939(08)09513-0

Article electronically published on July 8, 2008

\title{
ON BOUNDARY BLOW-UP PROBLEMS FOR THE COMPLEX MONGE-AMPÈRE EQUATION
}

\author{
SZYMON PLIŚ
}

(Communicated by Mei-Chi Shaw)

\begin{abstract}
We prove the $\mathcal{C}^{\infty}$ regularity for some complex Monge-Ampère equations with boundary data equal to $+\infty$.
\end{abstract}

\section{INTRODUCTION}

Cheng and Yau in $\mathrm{C}-\mathrm{Y}$ considered the following problem:

$$
\left\{\begin{array}{l}
\operatorname{det}\left(u_{p \bar{q}}\right)=g e^{K u} \text { in } \Omega, \\
\lim _{z \rightarrow z_{0}} u(z)=+\infty \text { for every } z_{0} \in \partial \Omega,
\end{array}\right.
$$

where $\Omega$ is a strictly pseudoconvex domain with $\mathcal{C}^{\infty}$ boundary (or some more general non-compact Kähler manifold), $K>0$ and $g \in \mathcal{C}^{\infty}(\bar{\Omega})$ is a positive function. They proved that problem (1.1) has a unique plurisubharmonic solution $u$ of class $\mathcal{C}^{\infty}$. This immediately gives a complete Kähler-Einstein metric on $\Omega$.

We will consider a more general problem than (1.1), i.e.

$$
\left\{\begin{array}{l}
\operatorname{det}\left(u_{p \bar{q}}\right)=g f(u) \text { in } \Omega, \\
\lim _{z \rightarrow z_{0}} u(z)=+\infty \text { for every } z_{0} \in \partial \Omega,
\end{array}\right.
$$

where $\Omega$ and $g$ are as above, and $f \in \mathcal{C}(\mathbb{R}) \cap \mathcal{C}^{\infty}((\tau,+\infty))$ for some $\tau \in \mathbb{R} \cup\{-\infty\}$ such that $f=0$ on $(-\infty, \tau)$ and $f^{\prime}>0$ on $(\tau,+\infty)$. We will show (see Theorem 2.1) the existence of a smooth solution for functions $f$ satisfying some technical conditions, which are fulfilled for example if $f(t)=e^{e^{e^{e^{t}}}}$ with $\tau=-\infty$ and if $f(t)=t^{p}$ for $p>n, f(t)=t^{n}+t^{n+1}$, and $f(t)=t^{n}(\log (t+2))^{2 n}$ with $\tau=0$.

This article is organized as follows. In section 2 we formulate the main theorem, and we give the proof of it in sections 3-5. In sections 6 and 7 we obtain something more about the case $f(t)=t^{p}$.

\section{MAIN THEOREM}

Let us first consider the following condition:

W1: the function

$$
\Psi(x)=\int_{x}^{\infty} \frac{d t}{F(t)^{1 /(n+1)}},
$$

Received by the editors November 6, 2007.

2000 Mathematics Subject Classification. Primary 32W20, 35B65.

Key words and phrases. Complex Monge-Ampère equation, blow up problem.

This research was partially supported by Polish grant MNiSW 3342/H03/2006/31.

(C) 2008 American Mathematical Society 
where $F$ is such that $F^{\prime}=f$, is well defined for $x$ large enough.

It is easy to check (see [G-P]) that condition W1 implies

$$
\lim _{x \rightarrow+\infty} \frac{F(x)^{n /(n+1)}}{f(x)}=0 .
$$

Ivarsson and Matero proved in I-M that if $\Omega=B=\{z:|z|<1\}, g$ depends only on $|z|, \tau=-\infty$ and $f$ satisfies condition W1, then problem (1.2) has a plurisubharmonic solution $u \in \mathcal{C}^{\infty}(B)$. For $\Omega$ strictly pseudoconvex with the boundary of class $\mathcal{C}^{\infty}$ Ivarsson proved in [I] that if $\tau=-\infty, g \equiv 1, f$ fulfills W1 and the condition $\frac{F(x) f^{\prime}(x)}{f^{2}(x)} \geq \frac{n-1}{n+1}$ for $x$ large enough, then problem (1.2) has a plurisubharmonic and locally Lipschitz solution $u$.

By $\mathrm{C}-\mathrm{K}-\mathrm{N}-\mathrm{S}]$ there exists a unique solution $w$ of

$$
\left\{\begin{array}{l}
w \in \mathcal{P} \mathcal{S H}(\Omega) \cap \mathcal{C}^{\infty}(\bar{\Omega}), \\
\operatorname{det}\left(w_{p \bar{q}}\right)=g \text { in } \Omega, \\
w=0 \text { on } \partial \Omega
\end{array}\right.
$$

Let us put

$$
\Phi(x)=\int_{x}^{\infty} \frac{d t}{f(t)^{1 / n}} \text { for } x>\tau ;
$$

then by the condition $\mathrm{W} 1$, it is well defined. Let $T=\left(\Phi^{-1}\left(\sup _{\Omega}(-w)\right),+\infty\right)$.

To formulate the main theorem we will also consider the following two conditions:

\section{W2:}

$$
\Phi(\tau)=\lim _{x \rightarrow \tau^{+}} \Phi(x)>\sup _{\Omega}(-w) .
$$

W3: There exist $\alpha, \beta, \gamma \geq 1$ such that on $T$ we have

$$
\begin{aligned}
& \alpha^{-1} f^{-\beta} \leq f^{\prime} \leq \alpha f^{\beta}, \\
& (\log f)^{\prime \prime} \leq \gamma(\log f)^{\prime 2} .
\end{aligned}
$$

Now we can formulate the main theorem:

Theorem 2.1. Let $\Omega, f$ and $g$ be as above. If $\mathrm{W} 1-\mathrm{W} 3$ are satisfied, then there exists a plurisubharmonic solution $u \in \mathcal{C}^{\infty}(\Omega)$ of the problem (1.2).

Note that if $\tau=-\infty$ or $f(\tau+x) \leq C x^{n}$ for $x$ in some interval $[0, \varepsilon]$, then condition $\mathrm{W} 2$ is satisfied. Otherwise, for $\varepsilon$ small enough it is satisfied in $\varepsilon \Omega=\{\varepsilon z: z \in \Omega\}$ if we also change $g$ to $g \circ S$, where $S(z)=\frac{z}{\varepsilon}$. Theorem 3.1 in [M] claims the existence of a smooth solution to a similar problem for the real Monge-Ampère equation without any condition on $f$ near $\tau$. We believe however that the proof only works if $f$ is replaced by $\lambda f$, where $\lambda$ is a constant such that the condition analogous to W2 holds. So it seems to be natural (in the complex and real cases) to assume something like W2. We will see that it enables us to construct a solution $u>\tau$.

We believe that assumption W3 in Theorem 2.1 is in fact superfluous. However the author was unable to prove a priori estimates without this condition. To see that many functions satisfy this condition, note that for positive and increasing $f$ and $x>1$ we have

$$
\liminf _{t \rightarrow+\infty} \frac{f^{\prime}(t)}{f(t)^{x}}=0 \geq \liminf _{t \rightarrow+\infty} \frac{(\log f(t))^{\prime \prime}}{(\log f(t))^{\prime x}}
$$


Also, if $f$ satisfy $W 1$, then

$$
\limsup _{t \rightarrow+\infty} \frac{f^{\prime}(t)}{f(t)^{n-1 / n}}=+\infty
$$

\section{Approximating Sequence}

In the same way as in $[\mathrm{M}$ (in the real case) we will define an increasing sequence of plurisubharmonic functions approximating the solution of problem (1.2). Let $\varphi=(-\Phi)^{-1} \circ w, \Omega_{k}=\left\{z \in \mathbb{C}^{n}: \varphi(z)<k\right\}$ (where $k$ is such that the set $\Omega_{k}$ is a strictly pseudoconvex domain of class $\mathcal{C}^{\infty}$ ) and let $u_{k}$ be the solution of the following Dirichlet problem:

$$
\left\{\begin{array}{l}
u \in \mathcal{P} \mathcal{S H} \cap \mathcal{C}^{\infty}\left(\bar{\Omega}_{k}\right), \\
\operatorname{det}\left(u_{p \bar{q}}\right)=g f(u) \text { in } \Omega_{k}, \\
u=k(=\varphi) \text { in } \partial \Omega_{k} .
\end{array}\right.
$$

The following lemma is an easy consequence of the comparison principle.

Lemma 3.1. We have $u_{k+1} \geq u_{k} \geq \varphi$.

Proof. Let $V=(-\Psi)^{-1}$. Then $V>0$ is smooth, $V^{\prime}=f^{1 / n} \circ V>0$ and $V^{\prime \prime}=$ $\frac{f^{\prime}}{n F^{(n-2) / n}} \circ V>0$. So $V$ is a smooth plurisubharmonic function, and we can compute

$$
\begin{gathered}
\operatorname{det}\left(\varphi_{p \bar{q}}\right)=\operatorname{det}\left(V^{\prime} \circ w w_{p \bar{q}}+V^{\prime \prime} \circ w w_{p} w_{\bar{q}}\right) \\
=g f \circ V \circ w+\frac{1}{n} g f^{1 / n} f^{\prime} \circ V \circ w w^{p \bar{q}} w_{p} w_{\bar{q}}>g f \circ \varphi .
\end{gathered}
$$

By the comparison principle $u_{k} \geq \varphi$, and from this (again using the comparison principle) we obtain $u_{k+1} \geq u_{k}$.

\section{A priori estimates}

In this section we shall prove that $u_{k}$ and $\triangle u_{k}$ are locally bounded, and our estimate will be independent of $k$.

Differentiating (the logarithm of) the equation $\operatorname{det}\left(u_{p \bar{q}}\right)=g f(u)$ twice we get the following formulas, which are very useful in the theory of regularity of the Monge-Ampère equation:

$$
\begin{gathered}
(\log g f(u))_{k}=u^{p \bar{q}} u_{k p \bar{q}} \\
(\log g f(u))_{k \bar{k}}=u^{p \bar{q}} u_{k \bar{k} p \bar{q}}-u^{p \bar{j}} u^{i \bar{q}} u_{k i \bar{j}} u_{\bar{k} p \bar{q}},
\end{gathered}
$$

where $k=1, \ldots, n$ and $\left(u^{p \bar{q}}\right)$ is the inverse transposed matrix of $\left(u_{p \bar{q}}\right)$. We will also use the differential operator $L=L_{k}=u_{k}^{p \bar{q}} \frac{\partial^{2}}{\partial z_{p} \partial \bar{z}_{q}}$. Functions $u_{k}$ are plurisubharmonic; hence $L$ is elliptic and we can apply the maximum principle.

In a similar way as in [I] (or in the real case in $[\mathrm{M}]$ ) we will prove the following lemma:

Lemma 4.1. There is a function $H \in \mathcal{P S H} \cap \mathcal{C}(\Omega)$, such that for every $u \in$ $\mathcal{P S H}(\Omega) \cap \mathcal{C}(\bar{\Omega})$, if $\operatorname{det}\left(u_{p \bar{q}}\right) \geq g f(u)$, then $u \leq H$. 
Proof. Let $W=(-\Psi)^{-1}$ and $H=W \circ(K w)$, where $K>0$ (small enough so that $H$ is well defined) will be specified later. Then $W>0$ is smooth, $W^{\prime}=F^{1 /(n+1)} \circ W>$ 0 and $W^{\prime \prime}=\frac{f}{(n+1) F^{(n-1) /(n+1)}} \circ W>0$. So $H$ is a smooth plurisubharmonic function, and we can compute

$$
\begin{gathered}
\operatorname{det}\left(H_{p \bar{q}}\right)=\operatorname{det}\left(W^{\prime} \circ(K w) K w_{p \bar{q}}+W^{\prime \prime} \circ(K w) K^{2} w_{p} w_{\bar{q}}\right) \\
=g K^{n} F^{n /(n+1)} \circ W \circ(K w)+\frac{1}{n+1} g K^{n+1} f \circ W \circ(K w) w^{p \bar{q}} w_{p} w_{\bar{q}} \\
=g f(H)\left(\frac{K^{n} F^{n /(n+1)}}{f} \circ H+K^{n+1} \frac{w^{p \bar{q}} w_{p} w_{\bar{q}}}{n+1}\right) .
\end{gathered}
$$

By (2.1), for $K$ small enough we have $\operatorname{det}\left(H_{p \bar{q}}\right) \leq g f(H)$. So by the comparison principle we obtain $u \leq H$.

Lemma 4.2. There exists a constant $C$ independent of $k$ (it may depend on $\Omega, f$ and $g$ ) such that

$$
\left|\nabla u_{k}\right| \leq C f^{1 / n}(k)
$$

Proof. Since $u_{k} \geq \varphi$, on $\partial \Omega_{k}$ we have (as in [I])

$$
f^{-1 / n}\left(u_{k}\right)\left|\nabla u_{k}\right|=\left|\nabla\left(\Phi \circ u_{k}\right)\right| \leq|\nabla(\Phi \circ \varphi)|=|\nabla w| .
$$

Let $r \in\{1, \ldots, 2 n\}$. To estimate $\left(u_{k}\right)_{x_{r}}$ let us consider the function $\eta=\left(u_{k}\right)_{x_{r}}+$ $K f^{1 / n}(k)|z|^{2}$. If $\eta$ attains a maximum on the boundary, then the proof is complete, so we may assume that $\eta$ attains maximum at $z_{0} \in \Omega_{k}$. We can also assume that $\left(u_{k}\right)_{x_{r}}\left(z_{0}\right) \geq 0$. By (4.1) and because $f$ is increasing, we have at $z_{0}$

$$
\begin{gathered}
L\left(\left(u_{k}\right)_{x_{r}}+K f^{1 / n}(k)|z|^{2}\right)=\left(\log \left(g f\left(u_{k}\right)\right)\right)_{x_{r}}+K f^{1 / n}(k) \sum_{p=1}^{n} u^{p \bar{p}} \\
\geq(\log g)_{x_{r}}+n K>0
\end{gathered}
$$

for $K$ large enough. By the maximum principle we obtain (4.3).

Lemma 4.3. If conditions $\mathrm{W} 1-\mathrm{W} 3$ are fulfilled, then there are constants $N$ and $C$ independent of $k$ such that

$$
\frac{\triangle u_{k}}{f\left(u_{k}\right)^{N}} \leq C \text { on } \Omega_{k} .
$$

Proof. Let $c_{0}, c_{1}, c_{2}, \ldots$ be constants depending only on $\Omega,\|w\|_{\mathcal{C}^{2,1}}, f$ and $g$. For simplicity in what follows we will write $u$ instead of $u_{k}, \Omega$ instead of $\Omega_{k}$ and $\eta$ instead of $\eta \circ u$ (or $\eta(u)$ ).

We will show that for $N$ big enough the function

$$
\Lambda=\max _{i \in\{1, \ldots, n\}} \lambda_{i} f^{-N} e^{|z|^{2}}
$$

where $\lambda_{i}$ are eigenvalues of the matrix $\left(u_{p \bar{q}}\right)$, is bounded . The proof will be divided into two parts: first we will estimate $\Lambda$ on the boundary and then inside. 
Part I: Function $\Lambda$ is bounded on boundary of $\Omega$. Let $z_{0} \in \partial \Omega$. After a holomorphic change of variables (see for example the proof of Lemma 1.3 in [C-K-N-S] ) we can assume that

$$
\begin{gathered}
z_{0}=0, \\
w=-x_{2 n-1}+a_{p \bar{q}} z_{p} \bar{z}_{q}+o\left(|z|^{2}\right) \text { for some matrix }\left(a_{p \bar{q}}\right)_{p, q=1}^{n}>0, \\
w_{p \bar{q}}=a_{p \bar{q}}=0 \text { for } p, q<n \text { such that } p \neq q .
\end{gathered}
$$

Then (as in [C-K-N-S]

$$
u_{p \bar{q}}=\frac{u_{x_{2 n-1}}}{w_{x_{2 n-1}}} w_{p \bar{q}} \text { for } p, q<n .
$$

For $l=1, \ldots, 2 n-2$ we consider

$$
w_{l}= \pm T_{l} u+\left(u_{x_{l}}\right)^{2}+\left(u_{x_{2 n}}\right)^{2}+A|z|^{2}-B x_{2 n-1},
$$

where $T_{l}=a \partial_{x_{l}}-b \partial_{x_{2 n-1}}, a=\rho_{x_{2 n-1}}$ and $b=\rho_{x_{l}}$. We have

$$
\begin{aligned}
& u^{p \bar{q}} u_{x_{2 n-1} \bar{q}}=2 u^{p \bar{q}} u_{n \bar{q}}+i u^{p \bar{q}} u_{x_{2 n} \bar{q}}=2 \delta_{p n}+i u^{p \bar{q}} u_{x_{2 n} \bar{q}}, \\
& u^{p \bar{q}} u_{x_{2 n-1} p}=2 u^{p \bar{q}} u_{p \bar{n}}-i u^{p \bar{q}} u_{x_{2 n} p}=2 \delta_{q n}-i u^{p \bar{q}} u_{x_{2 n} p} .
\end{aligned}
$$

Thus using (4.1) and the Schwarz inequality we can calculate

$$
\begin{aligned}
L w_{l}= & \pm u^{p \bar{q}}\left(a_{p \bar{q}} u_{x_{l}}-b_{p \bar{q}} u_{x_{2 n-1}}+a_{p} u_{x_{l} \bar{q}}-b_{p} u_{x_{2 n-1} \bar{q}}+a_{\bar{q}} u_{x_{n} p}-b_{\bar{q}} u_{x_{2 n-1} p}\right) \\
& \pm T_{l} \log (g f)+2 u_{x_{l}}(\log (g f))_{x_{l}}+2 u^{p \bar{q}} u_{p x_{l}} u_{\bar{q} x_{l}} \\
& +2 u_{x_{2 n}}(\log (g f))_{x_{2 n}}+2 u^{p \bar{q}} u_{p x_{2 n}} u_{\bar{q} x_{2 n}}+A \sum_{p} u^{p \bar{p}} \\
\geq & -c_{1}|\nabla u| \sum_{p} u^{p \bar{p}}-c_{2} \sum_{p} u^{p \bar{p}}-2 \sqrt{u^{p \bar{q}} a_{p} a_{\bar{q}}} \sqrt{u^{p \bar{q}} u_{x_{l} p} u_{x_{l} \bar{q}}} \\
& -2 \sqrt{u^{p \bar{q}} b_{p} b_{\bar{q}}} \sqrt{u^{p \bar{q}} u_{x_{2 n} p} u_{x_{2 n} \bar{q}}}-c_{3}-c_{4} \frac{f^{\prime}|\nabla u|}{f}-c_{5}|\nabla u|-4 \frac{f^{\prime}|\nabla u|^{2}}{f} \\
& +2 u^{p \bar{q}} u_{p x_{l}} u_{\bar{q} x_{l}}+2 u^{p \bar{q}} u_{p x_{2 n}} u_{\bar{q} x_{2 n}}+A \sum_{p} u^{p \bar{p}} .
\end{aligned}
$$

We have $\sum u^{p \bar{p}} \geq \frac{1}{f^{1 / n}(k)}$; hence (also using the inequality between arithmetic and geometric means and Lemma 4.2) for some $A<c_{6}\left(f^{2 / n}(k)+\sup _{\Omega} f^{\prime}\right)$ we obtain $L w_{l}>0$.

Let $S_{\varepsilon}$ be the connected component of the set $\left\{z \in \Omega: x_{2 n-1}<\varepsilon\right\}$ such that $0 \in \bar{S}_{\varepsilon}$. For well chosen (small enough and independent of $k$ and $\left.z_{0}\right) \varepsilon>0$ and for every $z \in \partial S_{\varepsilon}$ we have

$$
|z| \leq \frac{c_{7}}{\varepsilon} \sqrt{x_{2 n-1}}=c_{8} \sqrt{x_{2 n-1}}
$$

Then, since $T_{l} u=0$ on $\partial \Omega \cap \partial S_{\varepsilon}$, we have

$$
\pm T_{l} u \leq c_{9} x_{2 n-1} \text { on } \partial S_{\varepsilon}
$$

and by Lemma 4.2

$$
\left(u_{x_{r}}\right)^{2} \leq c_{10}|\nabla u|^{2}|z|^{2} \leq c_{11} f^{2 / n}(k) x_{2 n-1} \text { for } r \neq 2 n-1 .
$$


Hence choosing suitable $B<c_{12}\left(f^{2 / n}(k)+\sup _{\Omega} f^{\prime}\right)$ we obtain $w_{l} \leq 0$ on $\partial S_{\varepsilon}$. From the maximum principle $w_{l} \leq 0$ holds on the whole of $S_{\varepsilon}$. This implies that $w_{l x_{2 n-1}}<c_{13}\left(f^{2 / n}(k)+\sup _{\Omega} f^{\prime}\right)$. By Lemma 4.2 and condition W3 we obtain

$$
\left|u_{p \bar{n}}\right|<c_{14} f^{N} \text { for some } N>0 .
$$

In our coordinates at 0 we can write the Monge-Ampère equation in the form

$$
g f=\prod_{k} u_{k \bar{k}}-\sum_{p \neq n} u_{p \bar{n}} u_{n \bar{p}} \prod_{p \neq k \neq n} u_{k \bar{k}} .
$$

Solving this and using (4.4) we can calculate

$$
u_{n \bar{n}}=\sum_{p \neq n} \frac{u_{p \bar{n}} u_{n \bar{p}} w_{x_{2 n-1}}}{w_{p \bar{p}} u_{x_{2 n-1}}}-\prod_{k \neq n} \frac{f g u_{x_{2 n-1}}}{w_{k \bar{k}} u_{x_{2 n-1}}} .
$$

By Lemma 3.1 there exists a constant $\nu>0$ such that $f g>\nu$ on $\Omega$. So using the inequality between arithmetic and geometric means, we can estimate

$$
\triangle u \geq n \nu^{1 / n}
$$

and then from the Hopf Lemma there exists a constant $\mu>0$ such that $u_{x_{2 n-1}}>\mu$. Thus, using (4.4), (4.5) and (4.6) we conclude that

$$
\triangle u<c_{15} f^{N}
$$

Part II: Function $\Lambda$ is bounded in $\bar{\Omega}$. Assume that a maximum of the function $\Lambda$ is attained at $z_{0} \in \Omega$ (otherwise we are done). After a linear change of variables we can assume that at $z_{0}$ the matrix $\left(u_{p \bar{q}}\right)$ is diagonal and $u_{1 \overline{1}}=\max _{k \in\{1, \ldots, n\}} \lambda_{k}$. Let $h=u_{1 \overline{1}} f^{-N} e^{|z|^{2}}$. Then the function $h$ also attains a maximum at $z_{0}$ and $h\left(z_{0}\right)=\Lambda\left(z_{0}\right)$. From now on all formulas are assumed to hold at $z_{0}$. We may assume that

$$
h \geq C e^{\left|z_{0}\right|^{2}}
$$

for some (big enough) $C>0$, which will be specified later, and that

$$
\left|z_{0}\right|<\frac{1}{2 N}
$$

Then (again by the inequality between arithmetic and geometric means)

$$
\sum_{p} u^{p \bar{p}} \geq C f^{(N-1) /(n-1)} .
$$

Since $h_{p}=0$ for $p=1, \ldots, n$, we obtain

$$
u_{p 1 \overline{1}}=u_{1 \overline{1}}\left(N \frac{f^{\prime} u_{p}}{f}-\bar{z}_{p}\right) .
$$

We will estimate the following expression from below:

$$
L(\log h)=\frac{u^{p \bar{p}} u_{p \bar{p} 1 \overline{1}}}{u_{1 \overline{1}}}-\frac{u^{p \bar{p}} u_{p 1 \overline{1}} u_{\bar{p} 1 \overline{1}}}{u_{1 \overline{1}}^{2}}-N(\log f)^{\prime \prime} u^{p \bar{p}} u_{p} u_{\bar{p}}-N(\log f)^{\prime}+\sum_{1}^{n} u^{p \bar{p}} .
$$


Using (4.2), W3, (4.10), the generalized means inequality, (4.1) and the Schwarz inequality we compute

$$
\begin{gathered}
\frac{u^{p \bar{p}} u_{p \bar{p} 1 \overline{1}}}{u_{1 \overline{1}}}=\frac{(\log (g f))_{1 \overline{1}}}{u^{1 \overline{1}}}+u^{1 \overline{1}} u^{p \bar{p}} u^{q \bar{q}} u_{1 p \bar{q}} u_{\overline{1} \bar{p} q} \\
\geq \frac{(\log g)_{1 \overline{1}}}{u_{1 \overline{1}}}+(\log f)^{\prime}+\frac{(\log f)^{\prime \prime} u_{1} u_{\overline{1}}}{u_{1 \overline{1}}}+u^{p \bar{p}} \frac{u_{p 1 \overline{1}} u_{\bar{p} 1 \overline{1}}}{u_{1 \overline{1}}^{2}}+\sum_{p=2}^{n} \frac{u_{p 1 \overline{1}} u_{\bar{p} 1 \overline{1}}}{u_{p \bar{p}} u_{1 \overline{1}}^{2}}+\sum_{p=2}^{n} \frac{u_{p \bar{p} \overline{1}} u_{p \bar{p} 1}}{u_{p \bar{p}}{ }^{2} u_{1 \overline{1}}} \\
\geq-c_{16}+\frac{(\log f)^{\prime \prime} u_{1} u_{\overline{1}}}{u_{1 \overline{1}}}+u^{p \bar{p}} \frac{u_{p 1 \overline{1}} u_{\bar{p} 1 \overline{1}}}{u_{1 \overline{1}}^{2}} \\
+\sum_{p=2}^{n} u^{p \bar{p}}\left(N \frac{f^{\prime} u_{p}}{f}-\bar{z}_{p}\right)\left(N \frac{f^{\prime} u_{\bar{p}}}{f}-z_{p}\right)+\frac{1}{(n-1) u_{1 \overline{1}}}\left|(\log g f)_{1}-\frac{u_{1 \overline{1} 1}}{u_{1 \overline{1}}}\right|^{2} \\
\geq-c_{16}+\frac{(\log f)^{\prime \prime} u_{1} u_{\overline{1}}}{u_{1 \overline{1}}}+u^{p \bar{p}} \frac{u_{p 1 \overline{1}} u_{\bar{p} 1 \overline{1}}}{u_{1 \overline{1}}^{2}}+\left(N^{2}-1\right)(\log f)^{\prime 2} \sum_{p=2}^{n} u^{p \bar{p}} u_{p} u_{\bar{p}} \\
+(\log f)^{\prime 2} \sum_{p=2}^{n} u^{p \bar{p}} u_{p} u_{\bar{p}}+\frac{1}{4} \sum_{p=2}^{n} u^{p \bar{p}}-2 \sqrt{\sum_{p=2}^{n}\left((\log f)^{\prime}\right)^{2} u^{p \bar{p}} u_{p} u_{\bar{p}}} \sqrt[\sum_{p=2}^{n} u^{p \bar{p}} N^{2} z_{p} \bar{z}_{p}]{n} \\
-\frac{1}{4} \sum_{p=2}^{n} u^{p \bar{p}}+\sum_{p=2}^{n} u^{p \bar{p}}\left|z_{p}\right|^{2}+\frac{1}{(n-1) u_{1 \overline{1}}}\left|(\log g f)_{1}-\frac{u_{1 \overline{1} 1}}{u_{1 \overline{1}}}\right|^{2} .
\end{gathered}
$$

The inequality between arithmetic and geometric means and (4.8) gives us

$$
\begin{gathered}
\frac{u^{p \bar{p}} u_{p \bar{p} 1 \overline{1}}}{u_{1 \overline{1}}} \geq-c_{16}+u^{p \bar{p}} \frac{u_{p 1 \overline{1}} u_{\bar{p} 1 \overline{1}}}{u_{1 \overline{1}}^{2}}+\left(N^{2}-1\right)(\log f)^{\prime} \sum_{p=2}^{n} u^{p \bar{p}} u_{p} u_{\bar{p}} \\
-\frac{1}{4} \sum_{p=1}^{n} u^{p \bar{p}}+\frac{1}{(n-1) u_{1 \overline{1}}}\left|(\log g f)_{1}-\frac{u_{1 \overline{1} 1}}{u_{1 \overline{1}}}\right|^{2}+\frac{(\log f)^{\prime \prime} u_{1} u_{\overline{1}}}{u_{1 \overline{1}}} .
\end{gathered}
$$

Two cases may occur:

A)

$$
\left|u_{1}\right|<\frac{\left|(\log g)_{1}\right|+\left|z_{1}\right|}{(\log f)^{\prime}}
$$

Then by W3 we get that

$$
\left|u_{1}\right|<c_{17} f^{\alpha+1}
$$

Therefore by (4.7) and W3 we have

$$
-(\log f)^{\prime \prime} u^{1 \overline{1}} u_{1} u_{\overline{1}} \geq-c_{18} f^{4 \beta-N}
$$

B)

$$
\left|u_{1}\right| \geq \frac{\left|(\log g)_{1}\right|+\left|z_{1}\right|}{(\log f)^{\prime}}
$$


Then by (4.10) and W3 we have

$$
\begin{gathered}
\frac{1}{(n-1) u_{1 \overline{1}}}\left|(\log g f)_{1}-\frac{u_{1 \overline{1} 1}}{u_{1 \overline{1}}}\right|^{2}-(\log f)^{\prime \prime} u^{1 \overline{1}} u_{1} u_{\overline{1}} \\
=\frac{u^{1 \overline{1}} u_{1} u_{\overline{1}}}{n-1}\left|\frac{(\log g)_{1}-\overline{z_{1}}}{u_{1}(\log f)^{\prime}}+1-N\right|^{2}-(\log f)^{\prime \prime} u^{1 \overline{1}} u_{1} u_{\overline{1}} \\
\geq(\log f)^{\prime 2} u^{1 \overline{1}} u_{1} u_{\overline{1}}\left(\frac{(N-2)^{2}}{n-1}-\gamma\right) \geq c_{19} f^{2 \alpha-2}\left(\frac{(N-2)^{2}}{n-1}-\gamma\right) u^{1 \overline{1}} u_{1} u_{\overline{1}} .
\end{gathered}
$$

It is clear that in both cases for $N$ big enough, we obtain

$$
\frac{1}{(n-1) u_{1 \overline{1}}}\left|(\log g f)_{1}-\frac{u_{1 \overline{1}}}{u_{1 \overline{1}}}\right|^{2}-(N-1)(\log f)^{\prime \prime} u^{1 \overline{1}} u_{1} u_{\overline{1}} \geq-c_{20},
$$

so using (4.9), (4.12) and (4.7) we can conclude that for $C$ and $N$ big enough

$$
L(\log h)>0 .
$$

But this contradicts the fact that $h$ attains a maximum at $z_{0}$.

Note that in the above proof we obtained an a priori estimate on the boundary (Part I of proof) in the same way as in [C-K-N-S]. In Part II we used some ideas from [B2].

\section{Proof of Theorem 2.1}

Theorem 2.1 is an easy consequence of a priori estimates.

Proof of Theorem 2.1. Using Lemma 4.1 (for all domains $\Omega_{k}$ ), Lemma 3.1 and Lemma 4.3 we obtain that $u_{k}$ converges locally uniformly to some function $u$ and $\triangle u$ is locally bounded, so $u$ is in the Sobolev space $W^{2, p}$ for every $p>1$. The Convergence Theorem for the complex Monge-Ampère equation gives us the fact that $u$ is a solution of problem (1.2), so using Theorem 2.5 from [B1] we get $u \in \mathcal{C}^{2}(\Omega)$. Now Theorem 2.1 follows from Theorem 2.2 (again) from [B1].

Note that in Theorem 2.1 we can (without changing the proof) assume that $w \in \mathcal{C}^{2,1}(\bar{\Omega}) \cap \mathcal{C}^{\infty}(\Omega)$ instead of smoothness of $\partial \Omega$.

\section{A NECESSARY CONDITION}

Similar to the real case (see [L-M]) for the function $f(t)=t^{p}$, problem (1.2) has a solution if and only if $p>n$. It follows from the following theorem:

Theorem 6.1. If a solution of the problem

$$
\left\{\begin{array}{l}
u \in \mathcal{P S H} \cap \mathcal{C}(\Omega), \\
\operatorname{det}\left(u_{p \bar{q}}\right) \leq g f(u) \text { in } \Omega, \\
\lim _{z \rightarrow z_{0}} u(z)=+\infty \text { for } z_{0} \in \partial \Omega
\end{array}\right.
$$

exists, then the integral $\int \frac{1}{f^{1 / n}}$ converges at $+\infty$.

Proof (similar to $\underline{\mathrm{M}}$ ). We can assume that $\tau<0$ and that the function

$$
\mu(x)=\int_{0}^{x} \frac{d t}{f^{1 / n}(t)}
$$

is not bounded. Let $u$ be a solution of (6.1). Then for $A$ big enough the function $\phi_{A}=\mu^{-1} \circ(w+A)$ is well defined. In the same way as in Lemma 3.1, for the 
function $\varphi$ we obtain $\operatorname{det}\left(\phi_{A p \bar{q}}\right) \geq g f\left(\phi_{A}\right)$. From the comparison principle $u \geq \phi_{A}$. It is a contradiction with a free choice of large $A$.

\section{UNIQUENESS}

Ivarsson in [I] proved that if $\lim \sup _{t \rightarrow+\infty} \frac{-\Psi(t)}{\Psi^{\prime}(t)}<+\infty$, then the solution of problem (1.2) is unique. Using his method, we will also show this for $f(t)=t^{p}$ where $p>n$ (in this case $\lim _{t \rightarrow+\infty} \frac{-\Psi(t)}{\Psi^{\prime}(t)}=+\infty$ ).

Theorem 7.1. Let $u, v$ be plurisubharmonic solutions of problem (1.2). Assume $\tau=-\infty, \mathrm{W} 1$,

$$
\limsup _{t \rightarrow+\infty} \frac{-\Psi(t)}{t \Psi^{\prime}(t)}<+\infty
$$

and also

$$
(\log f(t))^{\prime} \geq \frac{n}{t+C}
$$

for some constant $C>0$ and $t \geq \inf _{\Omega} \min \{u, v\}$. Then $u \equiv v$.

Proof. First, we will prove that

$$
\lim _{z \rightarrow \partial \Omega} \frac{u(z)}{v(z)}=1 .
$$

Indeed, using (7.1), we obtain that there exist $D>0$ such that for $t$ big enough and $x \geq 1$ we have

so

$$
\frac{\partial}{\partial x}(\Psi(x t))=x \Psi^{\prime}(x t) \leq-D \Psi(x t)
$$

$$
\Psi(x t) \leq \Psi(t)\left(e^{D}\right)^{1-x} .
$$

Theorem 3.8 from [I-M] gives us

$$
\lim _{z \rightarrow \partial \Omega} \frac{\Psi(u(z))}{\Psi(v(z))}=1
$$

Thus, using (7.4) we can conclude that (17.3) follows.

Let $x \geq 1, u_{x}=x(u+C), v_{x}=x(v+C)$ and $f_{x}(t)=x^{n} f\left(\frac{t}{x}-C\right)$ for $t \in \mathbb{R}$. Note that $u_{x}, v_{x}>0$ and $\operatorname{det}\left(u_{x p \bar{q}}\right)=\operatorname{det}\left(v_{x p \bar{q}}\right)=g f_{x}(u)$ on $\Omega$. Inequality (7.2) means that $\frac{\partial f_{x}(t)}{\partial x} \geq 0$ for $t \in \mathbb{R}$, so $f_{x} \geq f_{1}$. Using (7.3) we obtain that the set $S=\left\{u_{x}<v_{1}\right\}$ is relatively compact in $\Omega$ for $x>1$. Then by the comparison principle $S$ is empty. Hence, because $\lim _{x \rightarrow 1+} u_{x}=u_{1}$, we have $u_{1} \geq v_{1}$, and in the same way we can obtain that $v_{1} \geq u_{1}$. Thus the theorem follows.

Corollary 7.2. Let $p>n$ and $f(t)=t^{p}$. Then there is exactly one plurisubharmonic solution of problem (1.2).

Proof. By Theorem 2.1 we know that there is a smooth solution of problem (1.2). The definition of $\varphi$ and Lemma 3.1 imply that our solution is positive. If $u$ is a positive solution we may suitably modify the function $f(t)=t^{p}$ for $t<\inf _{\Omega} u$ and get a positive, increasing function on $\mathbb{R}$. Hence from Theorem 7.1 we have only one positive solution. Now it is enough to prove that every solution is positive. To do this let us consider a strictly pseudoconvex domain $\tilde{\Omega}$, such that $\Omega$ is relatively 
compact in $\tilde{\Omega}$. Then on $\tilde{\Omega}$ there exists a positive plurisubharmonic solution $u$ of the equation

$$
\operatorname{det}\left(u_{p \bar{q}}\right)=\left(\sup _{\Omega} g\right) u^{p}
$$

and from the comparison principle $u$ is dominated from above by any solution of problem (1.2) which gives the statement.

\section{ACKNOWLEDGMent}

The author would like to express his gratitude to Z. Błocki for helpful discussions and advice during the work on this paper.

\section{REFERENCES}

[B1] Z. Błocki, On the regularity of the complex Monge-Ampère operator, Complex geometric analysis in Pohang (1997), 181-189, Contemp. Math., 222, Amer. Math. Soc., Providence, RI, 1999. MR1653050 (99m:32018)

[B2] Z. Błocki, Regularity of the degenerate Monge-Ampère equation on compact Kähler manifolds, Math. Z. 244 (2003), no. 1, 153-161. MR1981880(2004b:32065)

[C-K-N-S] L. Caffarelli, J. J. Kohn, L. Nirenberg, J. Spruck, The Dirichlet problem for non-linear second order elliptic equations, II: Complex Monge-Ampère, and uniformly elliptic equations, Comm. Pure Appl. Math. 38 (1985), 209-252. MR780073 (87f:35097)

[C-Y] S.-Y. Cheng, S.-Y. Yau, On the existence of a complete Kähler metric on non-compact complex manifolds and regularity of Fefferman's equation, Comm. Pure Appl. Math. 33 (1980), 507-544. MR575736 (82f:53074)

[G-P] F. Gladiali, G. Porru, Estimates for explosive solutions to p-Laplace equations, Progress in Partial Differential Equations (Pont-à-Mousson), Vol. 1, Pitman Res. Notes Math. Series, 383, Longman, Harlow (1998), 117-127. MR1628068 (2000h:35047)

[I] B. Ivarsson, Regularity and uniqueness of solutions to boundary blow-up problems for the complex Monge-Ampère operator, Bull. Polish Acad. Sci. Math. 54 (2006), 13-25. MR2270791 (2007g:32028)

[I-M] B. Ivarsson, J. Matero, The blow-up rate of solutions to boundary blow-up problems for the complex Monge-Ampère operator, Manuscripta Math. 120 (2006), no. 3, 325-345. MR 2243567(2007h:32058)

[L-M] A. C. Lazer, P. J. McKenna, On singular boundary value problems for the MongeAmpère operator, J. Math. Anal. Appl. 197 (1996), 341-362. MR1372183 (97c:35064)

[M] A. Mohammed, On the existence of solutions to the Monge-Ampère equation with infinite boundary values, Proc. Amer. Math. Soc. 138 no. 1 (2007), 141-149. MR 2280183

Institute of Mathematics, Cracow University of Technology, Warszawska 24, 31-155 Kraków, POLAND

E-mail address: splis@pk.edu.pl 